\title{
Explorations on Construction of Cognition-Aware Emergency Communication System
}

\author{
Haitao Wang \\ College of Communication Engineering \\ PLA Univ. of Sci. \& Tech. \\ Nanjing, China \\ Lihua Song \\ College of Command Information Systems \\ PLAUST \\ Nanjing, China \\ Hui Chen \\ College of Communication Engineering \\ PLA Univ. of Sci. \& Tech. \\ Nanjing, China
}

\author{
Li Yan \\ College of Communication Engineering \\ PLA Univ. of Sci. \& Tech. \\ Nanjing, China \\ Shicai Zhu \\ College of Communication Engineering \\ PLA Univ. of Sci. \& Tech. \\ Nanjing, China
}

\begin{abstract}
Cognitive Radio (CR) and Cognitive Network (CN) can optimize system objects by dynamically assigning network resource and adjusting network operations in terms of network environments and conditions. So these two cognitive technologies are very suitable for building emergency communication system to improve resource utilization ratio and enhancing dependability and availability of communication services. In this paper, background requirements of constructing cognitive emergency communication systems are expatiated and technical traits of CR and $\mathrm{CN}$ are introduced. Then, network architecture and model of cognitive emergency communication system are proposed. In addition, CPE and SAN are explained too. In the end, several application scenarios and future works of cognitive emergency communication network are discussed and prospected.
\end{abstract}

Keywords- Cognitive Radio; Cognitive Network; Emergency Communication System; Architecture; Cognitive Process Engine

\section{INTRODUCTION}

Emergency communication is a temporary and fast response special communication mechanism for coping with natural disasters or urgent cases by suing various communication techniques and resources [1]. The current deployment of emergency communication network, either the satellite shortwave or trunking communication system, mostly depends on the preset network infrastructure, with low reliability and lack of cognitive ability and adaptability, so it cannot make full use of scarce cyber source to optimize network performance. One of the two reasons is that internal network lacks timely and effective monitoring and response mechanism, so network units (including the host, network equipment and network protocol) cannot utilize time-varying cyber source dynamically and make reasonable, correct adjustments, and the other reason is the lack of enough coordination and communication between network elements, and they respond to network events just according to their own understanding of the information and capability. Obviously, such action is isolated and passive, not active coordination. In order to maximize the use of cyber source in the complex heterogeneous emergency network scene and ensure that emergency command communication can be carried out promptly and reliably, there is pressing need for us to upgrade the adaptability of current emergency communication network and allow network units to monitor network status actively, interact with each other and take appropriate action according to predetermined targets.

Cognitive Radio (CR) [2] is recognized as a new intelligent wireless communication technology, which improves the utilization rate of wireless spectrum through dynamic spectrum access and opportunistic spectrum access, in a wireless network environment with spectrum resource getting increasingly scarce. CR has the abilities of spectrum sensing, active learning and intelligent processing. It changes the real-time radio operating parameters and adjusts the internal state of the system through interaction with the working network environment and perception, understanding and active learning of the external environment so that the wireless device can automatically adapt to external wireless environment and the changing needs of themselves [3]. If we compare the traditional emergency communication system's defects with the advantages of cognitive radio technology, it's easy to discover that it will be very necessary to adopt the CR to establish a emergency communication system with cognitive ability in a scene with complex and changeful network environment and resource shortage. In that way, we could improve resource utilization efficiency of emergency communication network and enhance the intelligence, reliability and applicability of the emergency communication system in all kinds of network environment. In emergency scene, for instance, cognitive radio station could not only 
alter communication settings to reduce the channel interference and improve the service quality according to network conditions, but also make correct decisions via the network environment real time monitoring and analysis and adjust the network behavior to optimize the network overall performance.

\section{CONSTRUCTION OF COGNITIVE EMERGENCY COMMUNICATION SYSTEM}

\section{A. Network Architecture}

In view of the characteristics of emergency communication, a four-layer Cognitive Network supporting Emergency Communication (ECCN) is presented, as shown in figure 1. From the top to the bottom are heterogeneous network infrastructure layer, (software) adjustable/adaptive network layer and cognitive processing layer and application layer in the system structure of ECCN, described as follows:

- Network infrastructure layer contains the various network systems and communication equipments at the scene of event, and it' $s$ the platform network operates on.

- Adjustable network layer superimposes on the underlying network infrastructure, including Software Adjustable Network (SAN) unit [4], network state monitor and sensor. Adjustable network unit (e.g. cognitive radio station) is the execution unit of cognitive decisions, and it adopts practical operations on network systems and equipments based on the cognitive decision commands. Reconfigurable network is composed of network infrastructure layer and adjustable network layer (reconfiguration generally means that it can adapt to the network requirements by adjusting the operation parameters without any change of hardware).

- Cognitive processing layer is the core decisionmaking layer of ECCN. It receives emergency service requests in real time and gets access to the underlying network state information by network monitor and sensor. And then it analyzes the information of upper and lower layers to make decisions via Cognitive Processing Engine (CPE), and guides the operation of various lower adjustable network units. What's more, cognitive processing layer will also feedback information like network service level to the upper applications and users besides the control of decisions.

- The upper layer is the application target layer, and the targets of system task are proposed by the users or application needs which drive the behavior of emergency communication system through identification, adjustment and optimization. Without the guidance of target, network equipments may operate on different goals, which may cause undesired consequences. Finally, the cognitive network also includes providing an external network interface to interconnect with external cognitive/noncognitive network.

Figure 1. Cognitive emergency communication network's architecture

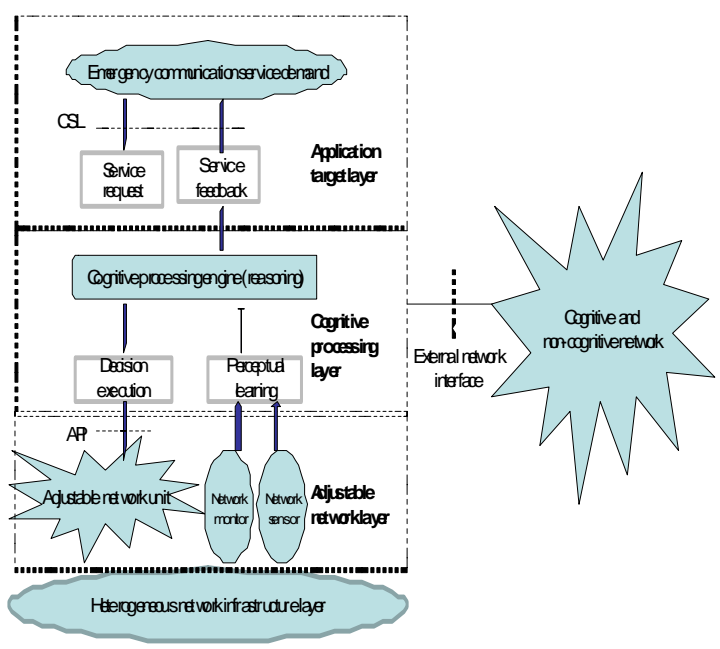

It is not difficult to see that the ECCN architecture comprises two control loops: one is the feedback control loop between the application target layer and the cognitive processing layer (the users make a request to the network for service -> cognitive processing engine does analysis and inference $->$ the network feed its service ability back to the users -> users adjust their application requirements); the other is the cognitive control loop between the cognitive processing layer and the adjustable network layer ( monitors and sensors transmit the information of network state and adjustable network unit to the cognitive processing layer -> the cognitive processing engine does analysis and makes decisions $->$ the cognitive processing layer sends the instructions of decision to the adjustable network unit to guide network's specific operation). The change of network state can be active or passive. And passive changes are unpredictable, such as the node's shift, addition and deletion and the changes of wireless channel environment; active changes turn the network to a desired state through adjustment and configuration of the network devices in a planned way. The network state information includes local information (such as BER, available link bandwidth and node residual energy etc.) and global information (such as the end-to-end delay and network connectivity). In the architecture of ECCN network, all cognitive network nodes constituting a cognitive emergency network synergistically, take appropriate action to meet the requirements of application and achieve the system targets according to cognitive decisions [5].

\section{B. Cognitive Processing Engine}


By unique Cognitive Specification Language (CSL), cognitive processing engine maps the system target to a form the lower cognitive process could understand, in order to guide the specific operant behavior of the adjustable network units, and something like extensible markup language (XML) can be employed [6]. Cognitive processing engine utilizes a variety of artificial intelligence, machine learning, decision support, adaptive algorithms for learning and inference, and makes the best decision according to the current network status information with the successful experience and knowledge combined, and then has the information of these successful decision-making stored in a database for later direct use for a similar situation. In network design stage we can determine learning and inference rule in advance according to the experience, but cognitive processing engine could modify the predetermined rules in the running stage on the basis of the current network status. Whatever choice of learning method, cognitive processes need to be able to quickly learn or converge to a solution, and when the state is changed the learning is still able to achieve a fast convergence. For the network whose environment often change, for example, the mobile wireless network, fast convergence is very important.

As cognitive emergency communication network must coordinate network node action on the basis of application demand to optimize the system overall target, cognitive processing engine carries out integrated intelligent management and global optimization of cyber source on the cognitive network nodes. And the information interaction and collaborative operation between the CPE of several nodes enable multiple independent cognitive nodes to integrate as a unified cognitive network eventually. The functional structure of CPE is as shown in Figure 2. CPE is a multi-functional software entity which uses the network state information and the information of each layer of the protocol stack to make analysis based on the strategy information provided by the library. Then it schedules resource use through calling the appropriate optimization mechanism and algorithm, and adjusts the parameters of each layer in the cross-layer protocol stack to obtain the best system settings matching the application demand. Subsequently, CPE observes behaviors of nodes and the result of network optimization, then sums up the experience through reasoning and learning, update strategy and stored in a strategy library. In addition, the CPE can also make decision to employ suitable channel resource and communication technology at the right time, which guarantees the quality of service for different users [5].

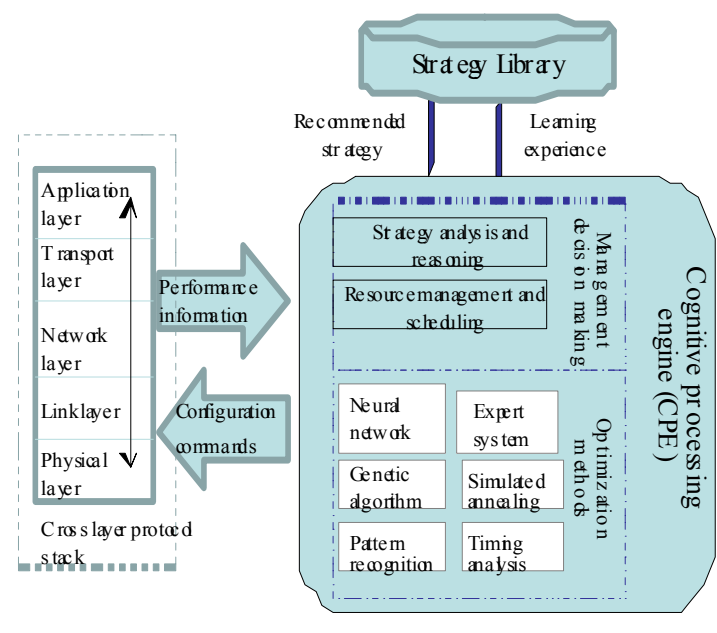

Figure 2. The functional structure of cognitive processing engine

CPE is modular and extensible, which may add the appropriate optimization and adjustment tools when needed, including neural networks, pattern recognition, genetic algorithm, expert system, timing analysis and Calman filter, etc. For example, genetic algorithm or simulated annealing method could be considered for use in the case that CPE will perform multi-dimensional optimization for large amounts of data. In order to deal with a large amount of historical data more effectively, it is necessary to carry out the information classification and clustering, using methods including neural network, time series analysis, etc. At the same time, in order to ensure that the operation of CPE is reliable, we must guarantee the quality of data for use in the decision-making process, and the Calman filter, Bayesian inference and statistical learning theory could be employed to deal with the uncertainty of reasoning and ensure the reliability of data.

\section{Software Adjustable Network}

Software adjustable network (SAN) is actually an independent research field, like the SDR design independent of cognitive radio. However, SAN needs to provide network interfaces which cognitive processing layer can understand and use. The interfaces should be flexible and extensible, which are similar to the application program interface (API) or an interface description language (IDL). SAN also includes the network elements that could be modified, and they can be used as policy control points (PCP) of the cognitive network. These network elements may include any internet object or element, and cognitive processing layer can operate each adjustable network element through the API. A simple example of software adjustable network (SAN) is a wireless network that supports directional antenna (antenna can search for receiving or transmit at different rotation angle). The wireless network which includes a network unit for regulating has the basic features of SAN. But it needs to be pointed out that it can be called 
cognitive network, only when adjusting the antenna direction is to serve the target of the system's cognitive behavior. Otherwise, it can only be called a wireless network using smart antenna, if you modify the antenna only in order to realize the link layer's partial target.

\section{ANALYSIS OF APPLICATION SCENARIOS}

The emergency communication system with cognitive ability designed in this paper can deal with resource shortage in an emergency through real time resource perception and adaptive management, which is applicable to a variety of emergency scenarios [7-8]. First of all, the wireless spectrum resource scarcity makes special allocation of emergency communication frequency band very difficult, and the limited spectrum cannot meet the needs of the rapidly growing emergency communication service demand. The emergency communication system with cognitive ability doesn't need to distribute the spectrum beforehand or negotiate communication frequency; it can improve emergency communication capacity by using idle spectrum dynamically. Secondly, cognitive emergency communication network can guarantee the quality of key service through the priority and the preemption right of spectrum usage in a condition with no network infrastructure. Thirdly, after an emergency incident, it will be time-consuming and laborconsuming to reconstruct the network according to the damage to infrastructure at the site, whereas cognitive emergency communication system can sense network dynamic changes of the environment in real time and realize adaptive resource management, to meet the fast and flexible support for emergency communication needs. Finally, it can set cognitive decision center node in the temporary emergency network organized by various departments on the scene in a case that public communications network paralyzes, and the emergency decision center of each sector can also be attached to the rear command and control center, with unified allocation and scheduling of emergency communication resources realize and the joint collaboration of network across different functional departments [12]. Take dealing with unexpected events in a city as an example, we'll describe the application of cognitive emergency communication network.

Emergency treatment is an important research topic of modern city management, and it is a premise that the emergency department personnel (police, fire fighting, medical staff) understands the situation rapidly, accurately after arriving at the scene for handling an emergency successfully. On the emergency scene, each of the rescue agencies usually tends to deploy their own emergency radio communication network, and will compete for the use of these scarce wireless resources, thus causing severe communication interference, thereby hampering rescue action. Therefore, we could upgrade the emergency wireless communication networks of various agencies to equip them with cognitive ability, in order to coordinate the emergency communication networks' operation of multiple agencies and deliver a variety of emergency information accurately and timely. For example, the cognitive radios carried by emergency personnel of various agencies are able to detect and collect active radio locations and transmitting frequency information through adaptive spectral perception, and optimize the use of scarce frequency resources through the dynamic spectrum access (DSA), thus to improve the spectrum utilization rate and avoid the communication interference between the rescue organizations to some extent. In addition, cognitive emergency communication system can realize adaptive adjustment according to the network environment change to ensure that the different users and applications use cyber source in accordance with its importance and to guarantee the quality of important service.

\section{CONCLUSION}

Cognitive radio and cognitive network is a hot technique that has aroused wide concern and been subjected to intensive research in the industry in recent years, and it can improve the spectrum utilization rate significantly and optimize the network performance. The thought of cognitive radio is applied to emergency communication field in this paper, and we provide the ideas of constructing a emergency communication system with cognitive ability; describe the network system structure, cognitive processing engine, adjustable network and other key components, and analyses several common application scenes. At present, the research on the emergency communication network with cognitive ability has just started; there are still many problems about technique and management to be solved. We must excavate the potential of emerging preponderant techniques including cognitive radio, cognitive network, ad hoc network, cooperative communication based on the realistic requirement, and establish an adaptive stereo emergency communication system with dynamic resource perception access, the combination of the space and the ground, the combination of the wired and the wireless, and the combination of the static and the dynamic to maximize the social service efficiency of emergency communications.

\section{ACKNOWLEDGMENT}

This paper is supported by NSFC (NO: 61072043).

\section{REFERENCES}

[1] Xueli Zhang, Rui Wang, Xiaolu Dong, etc, "Emergency communication new technology and system applications," Machinery Industry Press, Beijing, 2010.

[2] HAYKIN S, "Cognitive radio: Brain-empowered wireless communications [J]," IEEE Journal on Selected Areas in Communications, 2005, 23 (2): 201-220.

[3] P. Sutton, L. E. Doyle, and K. E. Nolan, "A Reconfigurable Platform for Cognitive Networks," Proc. CROWNCOM 2006, Mykonos Island, Greece, June, 2006:231-238.

[4] Ryan W. Thomas, Daniel H. Friend, Luiz A. DaSilv, "Cognitive Networks Adaptation and Learning to Achieve End-to-End performace objective," IEEE Communications Magazine, December 2006:51-57. 
[5] Jing Zhang, Hongbo Tang., "Emergency mobile communication system based on dynamic spectrum access," Telecommunications Science, 2008 (12) : 16-20.

[6] Chao Liu, Haitao Wang, "A new trend of the network development -cognitive network," Data communication, 2009 (2) : 1-5.
[7] P. Pawełczak, R. V. Prasad, L. Xia. Niemegeers, "Cognitive radio emergency networks - requirements and design," IEEE DySPAN 2005, Nov. 8-11, 2005, pp. 601-606.

[8] Gorcin.A , Arslan.H, "Public Safety and Emergency Case Communications: Opportunities from the Aspect of Cognitive Radio [C] ," DySPAN 2008. 3rd IEEE Symposium on, Chicago, USA, 14-17 Oct. 2008: 1-10. 